OPEN ACCESS

Edited by:

Mauro D'Onofrio,

Università degli Studi di Padova, Italy

Reviewed by:

Francoise Combes,

Observatoire de Paris, France

Giulia Rodighiero,

Università degli Studi di Padova, Italy

*Correspondence:

Wako Ishibashi

wako.ishibashi@physik.uzh.ch

Specialty section:

This article was submitted to

Milky Way and Galaxies,

a section of the journal

Frontiers in Astronomy and Space

Sciences

Received: 24 August 2017 Accepted: 02 October 2017

Published: 17 October 2017

Citation:

Ishibashi W (2017) How Quasar

Feedback May Shape the

Co-evolutionary Paths.

Front. Astron. Space Sci. 4:26.

doi: 10.3389/fspas.2017.00026

\section{How Quasar Feedback May Shape the Co-evolutionary Paths}

\author{
Wako Ishibashi* \\ Physik-Institut, University of Zurich, Zürich, Switzerland
}

Observations point toward some form of "co-evolutionary sequence," from dust-enshrouded starbursts to luminous unobscured quasars. Active galactic nucleus (AGN) feedback is generally invoked to expel the obscuring dusty gas in a blow-out event, eventually revealing the hidden central quasar. However, the physical mechanism driving AGN feedback, either due to winds or radiation, remains uncertain and is still a source of much debate. We consider quasar feedback, based on radiation pressure on dust, which directly acts on the obscuring dusty gas. We show that AGN radiative feedback is capable of efficiently removing the obscuring cocoon, and driving powerful outflows on galactic scales, consistent with recent observations. I will discuss how such quasar feedback may provide a natural physical interpretation of the observed evolutionary path, and the physical implications in the broader context of black hole-host galaxy co-evolution.

Keywords: active galactic nuclei, quasars, feedback, starbursts, galaxies

\section{INTRODUCTION}

Active galactic nuclei (AGN) and nuclear starbursts are observed to be intimately coupled, both fueled by the rapid infall of matter, possibly triggered by galaxy mergers (e.g., Alexander and Hickox, 2012, and references therein). A "co-evolutionary sequence," starting from dust-obscured starbursts (e.g., ultraluminous infrared galaxies or ULIRG) leading to unobscured optical quasars, has been widely discussed since Sanders et al. (1988). In most evolutionary scenarios, some form of AGN feedback is required to expel the obscuring gas and dust in a short-lived "blow-out" event.

Numerical simulations try to reproduce such an evolutionary sequence, with AGN feedback usually implemented by coupling a fixed fraction of the accretion luminosity to the surrounding medium (Di Matteo et al., 2005; Hopkins et al., 2005). However, the physical mechanism for AGN feedback is not specified, and in particular no explicit connection is made with the dust component (which is the fundamental parameter determining both the source visibility and the spectral energy distribution). On the observational side, there has been renewed interest in the topic, following the discovery of new populations of dust-reddened quasars at high redshifts (close to the peak epoch of both AGN and starburst activities), likely caught in the short-lived blow-out phase (e.g., Banerji et al., 2012, 2015).

Here we propose a physical mechanism that directly makes use of the dust in order to drive AGN feedback, and we briefly discuss how such AGN radiative feedback may naturally explain the observed co-evolutionary path (Ishibashi and Fabian, 2016b). 


\section{AGN RADIATIVE FEEDBACK: RADIATION PRESSURE ON DUST}

We consider AGN feedback driven by radiation pressure on dust. The ambient dusty gas is swept up into an outflowing shell, with the corresponding equation of motion given by:

$$
\frac{d}{d t}\left[M_{s h}(r) v\right]=\frac{L}{c}\left(1+\tau_{I R}-e^{-\tau_{U V}}\right)-\frac{G M(r) M_{s h}(r)}{r^{2}}
$$

where $L$ is the central luminosity, $M(r)$ is the total mass distribution, $M_{s h}(r)$ is the shell mass, and $\tau_{I R, U V}(r)=\frac{\kappa_{I R, U V} M_{s h}(r)}{4 \pi r^{2}}$ are the infrared (IR) and ultraviolet (UV) optical depths (Ishibashi and Fabian, 2015; Thompson et al., 2015). We recall that there are 3 distinct physical regimes depending on the optical depth of the medium: optically thick to both IR and UV, optically thick to UV but optically thin to IR (single scattering limit), and optically thin to UV. The associated shell column density is defined as:

$$
N_{s h}(r)=\frac{M_{s h}(r)}{4 \pi r^{2} m_{p}}
$$

where $m_{p}$ is the proton mass.

By equating the outward force due to radiation pressure to the inward force due to gravity, we can define a critical luminosity, $L_{E}^{\prime}$, which may be considered as a generalized form of the effective Eddington luminosity. We recall that the effective Eddington luminosity for dusty gas is distinct from the standard Eddington luminosity relevant for ionized gas (Fabian et al., 2006). The corresponding effective Eddington ratio is then given by:

$$
\Gamma=\frac{L}{L_{E}^{\prime}}=\frac{L r^{2}}{c G M(r) M_{s h}(r)}\left(1+\tau_{I R}-e^{-\tau_{U V}}\right)
$$

The effective Eddington ratios in the 3 optical depth regimes are respectively given by:

$$
\begin{aligned}
\Gamma_{I R} & =\frac{\kappa_{I R} L}{4 \pi G c M(r)} ; \Gamma_{S S}=\frac{L}{4 \pi G c m_{p} M(r) N_{s h}(r)} ; \\
\Gamma_{U V} & =\frac{\kappa_{U V} L}{4 \pi G c M(r)}
\end{aligned}
$$

Figure 1 shows the effective Eddington ratio as a function of radius, and its dependence on the underlying physical parameters. The effective Eddington ratio basically corresponds to the ratio of the radiative force to the gravitational force, and must exceed unity for an outflowing shell (the standard Eddington limit does not apply here). From Figure 1, we see that an increase in the shell mass leads to a lower Eddington ratio in the single scattering regime (cyan curve); while in the IR-optically thick and UV-optically thin regimes, $\Gamma_{I R}$ and $\Gamma_{U V}$ are independent of the shell mass (cf. Equation 4), hence the cyan and black curves overlap at small and large radii. In contrast, enhanced opacities (e.g., due to a higher dust-to-gas ratio) lead to higher Eddington ratios in the IR-optically thick and UV-optically thin regimes (magenta curve); whereas in the single scattering regime, $\Gamma_{S S}$ is independent of the opacity (cf.

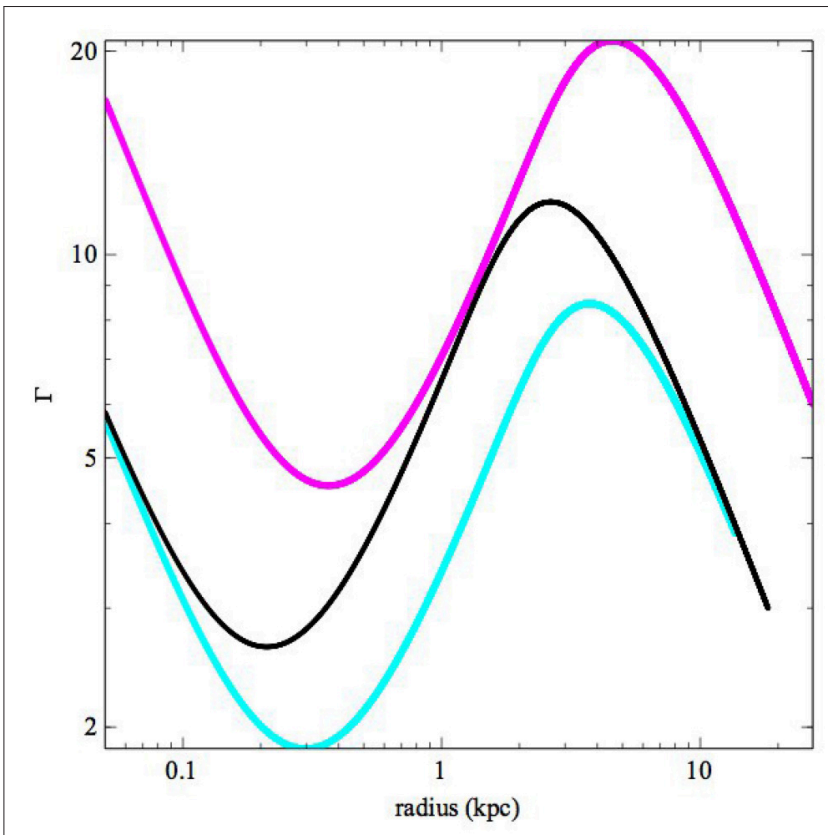

FIGURE 1 | Effective Eddington ratio as a function of radius: $L=5 \times$ $10^{46} \mathrm{erg} / \mathrm{s}, M_{\text {sh }}=5 \times 10^{8} M_{\odot}, f_{d g}=1 / 150$ (black, fiducial);

$L=5 \times 10^{46} \mathrm{erg} / \mathrm{s}, M_{s h}=1 \times 10^{9} M_{\odot}, f_{d g}=1 / 150$ (cyan);

$L=5 \times 10^{46} \mathrm{erg} / \mathrm{s}, M_{s h}=5 \times 10^{8} M_{\odot}, f_{d g}=1 / 50$ (magenta).

Equation 4), and thus the magenta and black curves overlap at intermediate radii. We note that there are two interesting trends in the IR-optically thick regime:

1. The effective Eddington ratio is independent of the shell column density (or shell mass).

2. The effective Eddington ratio directly scales with the opacity, and hence dust-to-gas ratio (as $\kappa_{I R} \propto f_{d g}$ ).

The first point implies that even dense material can potentially be disrupted; while the second point implies that the more dusty gas is more easily ejected (Ishibashi and Fabian, 2016b).

\section{DUST OBSCURATION AND BLOW-OUT}

In physical terms, dusty gas surrounding the central source absorbs UV radiation and re-emits in the IR band. If the reprocessed IR photons remain trapped in the nuclear regions, the system is effectively in the IR-optically thick regime, where the Eddington ratio is independent of the column density (Equation 4). Such conditions are likely reached in the nuclear regions of ULIRG-like systems, characterized by high densities and high dust-to-gas fractions. This leads to both higher IR optical depth and higher IR-Eddington ratio, which combine to facilitate the blow-out of dusty gas.

In Figure 2 (left panel) we plot the temporal evolution of the shell column density for different values of the central luminosity. We see that the obscuration falls off with time, with the decline being more rapid for brighter sources. In fact, an increase in the 

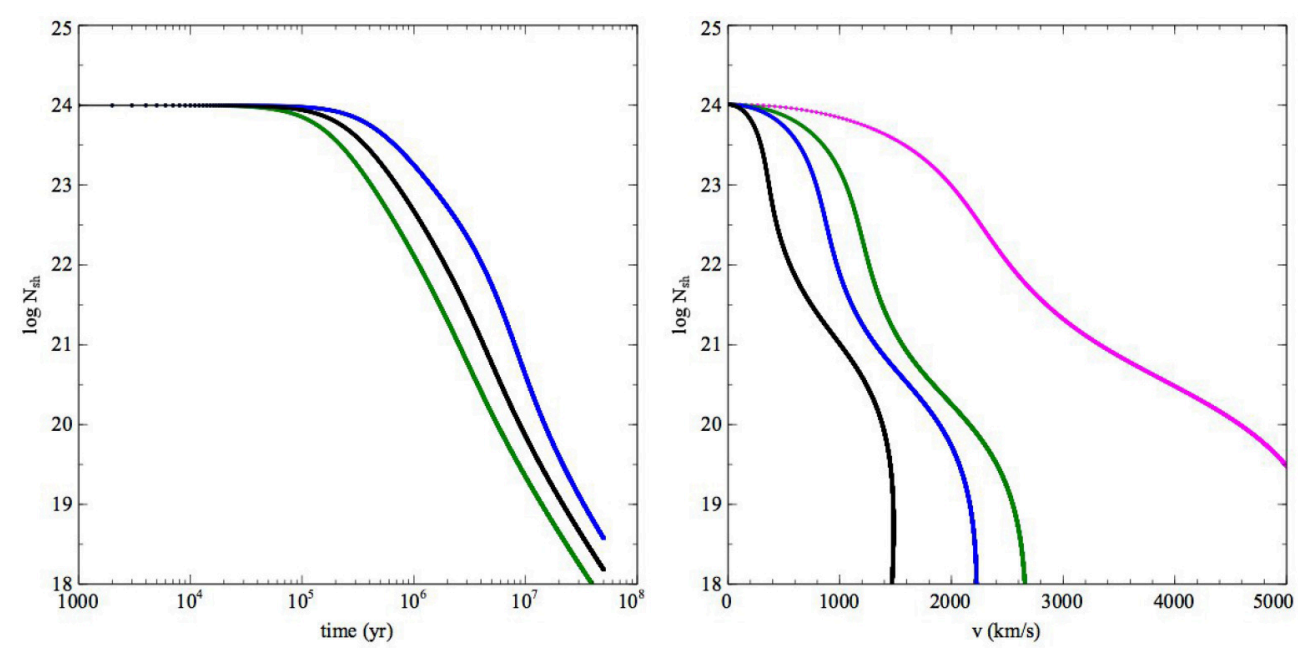

FIGURE 2 | Left: shell column density vs. time for variations in luminosity $\left(N_{s h, 0}=10^{24} \mathrm{~cm}^{-2}, f_{d g}=1 / 150\right): L=3 \times 10^{46} \mathrm{erg} / \mathrm{s}\left(\right.$ blue), $L=5 \times 10^{46} \mathrm{erg} / \mathrm{s}($ black), $L=1 \times 10^{47} \mathrm{erg} / \mathrm{s}$ (green). Right: shell column density vs. velocity for variations in the dust-to-gas ratio $\left(L=2 \times 10^{46} \mathrm{erg} / \mathrm{s}, N_{S h, 0}=10^{24} \mathrm{~cm}-2\right): f_{d g}=1 / 150($ black$)$, $f_{d g}=1 / 50$ (blue), $f_{d g}=1 / 30$ (green). High-luminosity case: $L=10^{47} \mathrm{erg} / \mathrm{s}, f_{d g}=1 / 50$ (magenta).

luminosity leads to an increase in the effective Eddington ratio in all 3 optical depth regimes, and thus efficient acceleration, and resulting high velocity. The right panel in Figure 2 shows the shell column density as a function of velocity for different values of the dust-to-gas ratio. We observe that a given column is accelerated to higher velocities for larger dust-to-gas fractions: at a given time, the column density is lower for higher dust-togas ratios. Indeed, an increase in the dust-to-gas ratio leads to enhanced IR opacity, which in turn leads to a higher Eddington ratio in the IR-optically thick regime. Thus the more dusty gas (which provides much of the obscuration) is preferentially ejected by radiative feedback, and therefore the central quasar has a natural tendency to remove its own obscuring cocoon and reveal itself. We have previously discussed how such AGN-driven dusty outflows may propagate on larger scales beyond the host galaxy, and contribute to the enrichment of the circum-galactic medium (Ishibashi and Fabian, 2016a).

The model results can be compared with observational samples of the recently uncovered populations of dust-reddened quasars and dust-obscured galaxies (Assef et al., 2015; Banerji et al., 2015; Zakamska et al., 2016). These sources are likely observed close to the point of ejecting their obscuring cocoon, and thus may represent the long-sought "sources in transition" (as discussed in Ishibashi and Fabian, 2016b).

\section{THE DUAL ROLE OF DUST: HIDING AND REVEALING THE CENTRAL QUASAR}

The large amounts of gas and dust surrounding the nuclear source provide potential fuel for the central black hole, but that same accreting material is also responsible for significant obscuration. Although AGN feedback is generally invoked to clear the obscuring gas and dust in co-evolutionary scenarios, the actual physical mechanism remains uncertain, with no clear connection to the dust component. In our picture, AGN radiative feedback directly acts on the dusty gas, which forms the obscuring medium. Large amounts of dust imply heavy obscuration, but also powerful feedback, suggesting a causal link between dust obscuration and blow-out. In this scenario, dust plays a dual role: it is responsible for both hiding, but eventually revealing the central quasar.

An important requirement in our model is the presence of dust, since the whole AGN feedback process relies on radiation pressure on dust. The large amount of dust required in order to sustain feedback may be provided by supernovae in the starburst phase. In fact, recent observations indicate that significant quantities of dust can be produced in core-collapse supernovae (Owen and Barlow, 2015; Wesson et al., 2015). Continued star formation in the starburst is beneficial, as it helps keeping the ambient medium dusty, and thus supporting the overall AGN feedback process. Stellar feedback likely disrupts the starburst activity, but the nuclear gas reservoir may also be replenished by re-accretion episodes. Furthermore, we previously suggested that star formation (with subsequent supernova explosions and associated dust release) may be triggered within the AGN feedback-driven outflow itself (Ishibashi and Fabian, 2012). Interestingly, the first direct detection of star formation occurring inside a galactic outflow has now been observationally confirmed (Maiolino et al., 2017), nicely supporting our model predictions. In our framework, AGN feedback and starburst phenomena are intrinsically coupled through the production of dust in supernova explosions, leading to a natural interpretation of the co-evolutionary path.

\section{AUTHOR CONTRIBUTIONS}

The author confirms being the sole contributor of this work and approved it for publication. 


\section{REFERENCES}

Alexander, D. M., and Hickox, R. C. (2012). What drives the growth of black holes?. New Astron. Rev. 56, 93-121. doi: 10.1016/j.newar.2011.11.003

Assef, R. J., Eisenhardt, P. R. M., Stern, D., Tsai, C.-W., Wu, J., Wylezalek, D., et al. (2015). Half of the most luminous quasars may be obscured: investigating the nature of WISE-selected hot dust-obscured galaxies. Astrophys. J. 804:27. doi: 10.1088/0004-637X/804/1/27

Banerji, M., Alaghband-Zadeh, S., Hewett, P. C., and McMahon, R. G. (2015). Heavily reddened type 1 quasars at $\mathrm{z}>2$ - I. Evidence for significant obscured black hole growth at the highest quasar luminosities. Mon. Not. R. Astron. Soc. 447, 3368-3389. doi: 10.1093/mnras/stu2649

Banerji, M., McMahon, R. G., Hewett, P. C., Alaghband-Zadeh, S., GonzalezSolares, E., Venemans, B. P., et al. (2012). Heavily reddened quasars at $\mathrm{z} \sim 2$ in the UKIDSS Large Area Survey: a transitional phase in AGN evolution. Mon. Not. R. Astron. Soc. 427, 2275-2291. doi: 10.1111/j.1365-2966.2012.22099.x

Di Matteo, T., Springel, V., and Hernquist, L. (2005). Energy input from quasars regulates the growth and activity of black holes and their host galaxies. Nature 433, 604-607. doi: 10.1038/nature03335

Fabian, A. C., Celotti, A., and Erlund, M. C. (2006). Radiative pressure feedback by a quasar in a galactic bulge. Mon. Not. R. Astron. Soc. 373, L16-L20. doi: 10.1111/j.1745-3933.2006.00234.x

Hopkins, P. F., Hernquist, L., Cox, T. J., Di Matteo, T., Martini, P., Robertson, B., et al. (2005). Black holes in galaxy mergers: evolution of quasars. Astrophys. J. 630, 705-715. doi: 10.1086/432438

Ishibashi, W., and Fabian, A. C. (2012). Active galactic nucleus feedback and triggering of star formation in galaxies. Mon. Not. R. Astron. Soc. 427, 2998-3005. doi: 10.1111/j.1365-2966.2012.22074.x

Ishibashi, W., and Fabian, A. C. (2015). AGN feedback: galactic-scale outflows driven by radiation pressure on dust. Mon. Not. R. Astron. Soc. 451, 93-102. doi: $10.1093 / \mathrm{mnras} / \mathrm{stv} 944$

Ishibashi, W., and Fabian, A. C. (2016a). AGN-starburst evolutionary connection: a physical interpretation based on radiative feedback. Mon. Not. R. Astron. Soc. 463, 1291-1296. doi: 10.1093/mnras/stw2063
Ishibashi, W., and Fabian, A. C. (2016b). The connection between AGN-driven dusty outflows and the surrounding environment. Mon. Not. R. Astron. Soc. 457, 2864-2870. doi: 10.1093/mnras/stw182

Maiolino, R., Russell, H. R., Fabian, A. C., Carniani, S., Gallagher, R., Cazzoli, S., et al. (2017). Star formation inside a galactic outflow. Nature 544, 202-206. doi: 10.1038/nature21677

Owen, P. J., and Barlow, M. J. (2015). The dust and gas content of the crab nebula. Astrophys. J. 801:141. doi: 10.1088/0004-637X/801/2/141

Sanders, D. B., Soifer, B. T., Elias, J. H., Madore, B. F., Matthews, K., Neugebauer, G., et al. (1988). Ultraluminous infrared galaxies and the origin of quasars. Astrophys. J. 325, 74-91. doi: 10.1086/165983

Thompson, T. A., Fabian, A. C., Quataert, E., and Murray, N. (2015). Dynamics of dusty radiation-pressure-driven shells and clouds: fast outflows from galaxies, star clusters, massive stars, and AGN. Mon. Not. R. Astron. Soc. 449, 147-161. doi: 10.1093/mnras/stv246

Wesson, R., Barlow, M. J., Matsuura, M., and Ercolano, B. (2015). The timing and location of dust formation in the remnant of SN 1987A. Mon. Not. R. Astron. Soc. 446, 2089-2101. doi: 10.1093/mnras/stu2250

Zakamska, N. L., Hamann, F., Pâris, I., Brandt, W. N., Greene, J. E., Strauss, M. A., et al. (2016). Discovery of extreme [O III] $\lambda 5007 \AA ̊$ outflows in high-redshift red quasars. Mon. Not. R. Astron. Soc. 459, 3144-3160. doi: 10.1093/mnras/stw718

Conflict of Interest Statement: The author declares that the research was conducted in the absence of any commercial or financial relationships that could be construed as a potential conflict of interest.

The reviewer GR and handling Editor declared their shared affiliation.

Copyright $\odot 2017$ Ishibashi. This is an open-access article distributed under the terms of the Creative Commons Attribution License (CC BY). The use, distribution or reproduction in other forums is permitted, provided the original author(s) or licensor are credited and that the original publication in this journal is cited, in accordance with accepted academic practice. No use, distribution or reproduction is permitted which does not comply with these terms. 